\title{
Trascendencia de los exosomas en la cardiología: heraldos de la cardioprotección
}

\author{
Significance of exosomes in cardiology: heralds of cardioprotection \\ Miguel Arroyo-Campuzano* y Cecilia Zazueta \\ Departamento de Biomedicina Cardiovascular, Instituto Nacional de Cardiología Ignacio Chávez, Ciudad de México, México
}

\begin{abstract}
Resumen
Los exosomas tienen un papel clave en la comunicación intercelular. Debido a sus múltiples interacciones, estas estructuras cumplen con el papel de «mensajeros" de forma dinámica, transportando su contenido a células blanco específicas y generando nuevas señales celulares. En este artículo se describen algunas de las proteínas, lípidos y ácidos nucleicos que son transportados por estas vesículas y que se han relacionado con cardioprotección, con la finalidad de proporcionar información y generar interés sobre la relevancia de los exosomas como posibles blancos diagnósticos y terapéuticos.
\end{abstract}

Palabras clave: Exosomas. Carga exosomal. Cardioprotección. Proteínas cardioprotectoras.

\begin{abstract}
Exosomes have a key role in intercellular communication. Due to their multiple interactions, these structures fulfill the role of "messengers" in a dynamic way, transporting their content to target-specific cells and generating new cellular signals. This article describes some of the proteins, lipids and nucleic acids that are transported by these vesicles and that have been related to cardioprotection, in order to provide information and generate interest in the relevance of exosomes as possible diagnostic and therapeutic targets.
\end{abstract}

Key words: Exosomes. Exosomal cargo. Cardioprotection. Cardioprotective proteins.

\section{Introducción}

Las células poseen un complejo sistema de tráfico vesicular que organiza tanto el transporte de proteínas y moléculas solubles a través del citoplasma, como de moléculas que son secretadas y que constituyen la base de la comunicación intercelular. En enfermedades cardiovasculares la secreción de dichas moléculas refleja la desregulación en la homeostasis celular, así como la disfunción celular y tisular, pero también pueden ser señales dirigidas a disminuir el daño celular ${ }^{1}$.

En este sentido, las vesículas extracelulares (VE), en particular los exosomas (EXO), cumplen una función paracrina, ya que al ser liberados al medio extracelular inducen respuestas antiapoptósicas, angiogénicas, inmunomoduladoras y antifibróticas ${ }^{2}$. Los tipos celulares descritos como fuentes potenciales de EXO con propiedades cardioprotectoras son las células madre mesenquimales,

\section{Correspondencia:}

*Miguel Arroyo-Campuzano

E-mail: arroyocampuzanomiguel@gmail.com
Fecha de recepción: 05-07-2020

Fecha de aceptación: 10-11-2020

DOI: $10.24875 /$ ACM.20000335
Disponible en internet: 23-02-2021 Arch Cardiol Mex. 2021;91(1):105-113 www.archivoscardiologia.com

(c) 2020 Instituto Nacional de Cardiología Ignacio Chávez. Publicado por Permanyer. Este es un artículo open access bajo la licencia CC BY-NC-ND (http://creativecommons.org/licenses/by-nc-nd/4.0/). 
cardiacas, embrionarias y hematopoyéticas ${ }^{3}$. Los EXO son nanocuerpos lipídicos generados por una doble endocitosis membranal, que se convierten en biocontenedores que transfieren biomoléculas a otras células (proteínas, lípidos, ácido ribonucleico [ARN] y ácido desoxirribonucleico [ADN] no codificante), modificando la función de las células receptoras ${ }^{2,3}$.

Los ensayos preclínicos en cardioprotección utilizando EXO son alentadores, sin embargo, aún se requiere generar más información para proponer que los efectos mediados por EXO estén directamente relacionados con las moléculas que transportan.

En esta revisión se describen los hallazgos sobre la eficacia cardioprotectora que tienen diferentes moléculas que son transportadas por los EXO, como las caveolinas (Cav), proteínas de choque térmico (HSP, heat shock proteins) y los micro ARN (miARN, miR), así como algunas vías de señalización en las que participan estas moléculas. Se espera generar interés en la relevancia del estudio de los EXO en cardioprotección, motivar el desarrollo de más ensayos preclínicos y dar avance hacia estudios clínicos.

\section{Generalidades de los exosomas}

\section{Características de los exosomas}

Los EXO son las VE más pequeñas, con un tamaño entre 30-150 nm y una densidad de 1.10-1.14 g/ml ${ }^{4}$. Se considera que participan en la remoción de proteínas de membrana, además de que su presencia en diferentes tejidos y fluidos corporales sugiere un papel fisiológico y patológico importante en la comunicación intercelular y/o entre órganos ${ }^{5}$. Entre las proteínas que se eliminan a través de esta vía se encuentran proteínas de la membrana plasmática (MP), como el receptor 1 de transferrina y componentes de «balsas lipídicas» como las tetraspaninas CD9, CD63 y CD814. Estas proteínas se organizan a lo largo de la membrana en regiones específicas de los EXO y se consideran sus biomarcadores más comunes ${ }^{6}$. Los EXO pueden tener diferente origen celular, por ejemplo: hematopoyético, de células epiteliales, células neurogliales y células cancerosas $^{7}$; por tanto, dependiendo de su origen celular, los EXO suelen recibir diferentes nombres, como: cardiosomas, vexosomas, prostasomas o tolerosomas ${ }^{8}$. Los EXO se encuentran en diferentes tipos de fluidos corporales, incluidos sangre, saliva, orina, semen, líquido amniótico, leche materna, líquido cefalorraquídeo, bilis, liquido de ascitis $^{9}$ y líquido pericárdico ${ }^{10}$.

\section{Biogénesis de los exosomas}

Inicialmente la MP debe sufrir un proceso de endocitosis; por macropicnocitosis, dependiente de proteínas como la clatrina, la flotilina, la GEEC (glycosylphosphotidylinositol-anchored protein enriched early endosomal compartments) o mediada por caveolas ${ }^{11}$. Al compartimento internalizado se le conoce como endosoma temprano, el cual madura en endosoma tardío y acumula vesículas intraluminales (VIL), por lo que también recibe el nombre de endosoma multivesicular (EMV). Las VIL se forman por invaginación de la membrana del EMV, internalizando proteínas, ácidos nucleicos, lípidos y citosol12 (Fig. 1).

Hasta el momento se han sugerido dos principales rutas para la formación de VIL. La primera se conoce como vía dependiente del complejo endosomal de clasificación requerido para el transporte (ESCRT, endosomal sorting complex required for transport), que es un complejo compuesto por cinco estructuras multiproteicas (ESCRT-0, ESCRT-I, ESCRT-II, ESCRT-III más el complejo Vps4), las cuales trabajan en coordinación para facilitar la gemación vesicular y la selección de la carga ${ }^{6}$. Al finalizar el proceso, los complejos ESCRT y moléculas relacionadas regresan al citosol, para su reutilización ${ }^{13}$.

La segunda vía implica a la esfingomielinasa neutral (nSMase2) $^{14}$, la cual genera ceramida a partir de esfingomielina. Se ha demostrado que la ceramida induce la unión de microdominios en dominios más grandes, promoviendo la formación de «brotes» en la membrana y la importancia de esta vía radica en que la expresión a la baja de la nSMase2 reduce la liberación de EXO'${ }^{12}$.

\section{Contenido exosomal}

La carga exosomal presenta características comunes con otros tipos de VE, sin embargo, gracias a diversos análisis se han identificado componentes más específicos $^{15}$. Incluso se ha determinado que esto depende del tipo celular del cual son secretados ${ }^{16}$. En la tabla 1 se enlistan algunos componentes de la estructura y contenido exosomal.

- Proteínas: los EXO se destacan por contener proteínas membranales, citosólicas y de la matriz extracelular de aproximadamente 4,400 clases diferentes ${ }^{6}$, sin embargo, contienen pocas proteínas del complejo de Golgi, del retículo endoplásmico, del núcleo y de mitocondrias ${ }^{16}$. Las proteínas mayormente reportadas son estructurales, como actina, cofilina-1 y tubulina, HSP, integrinas, enzimas metabólicas, tetraspaninas, proteínas relacionadas con el tráfico de vesículas como TSG101 


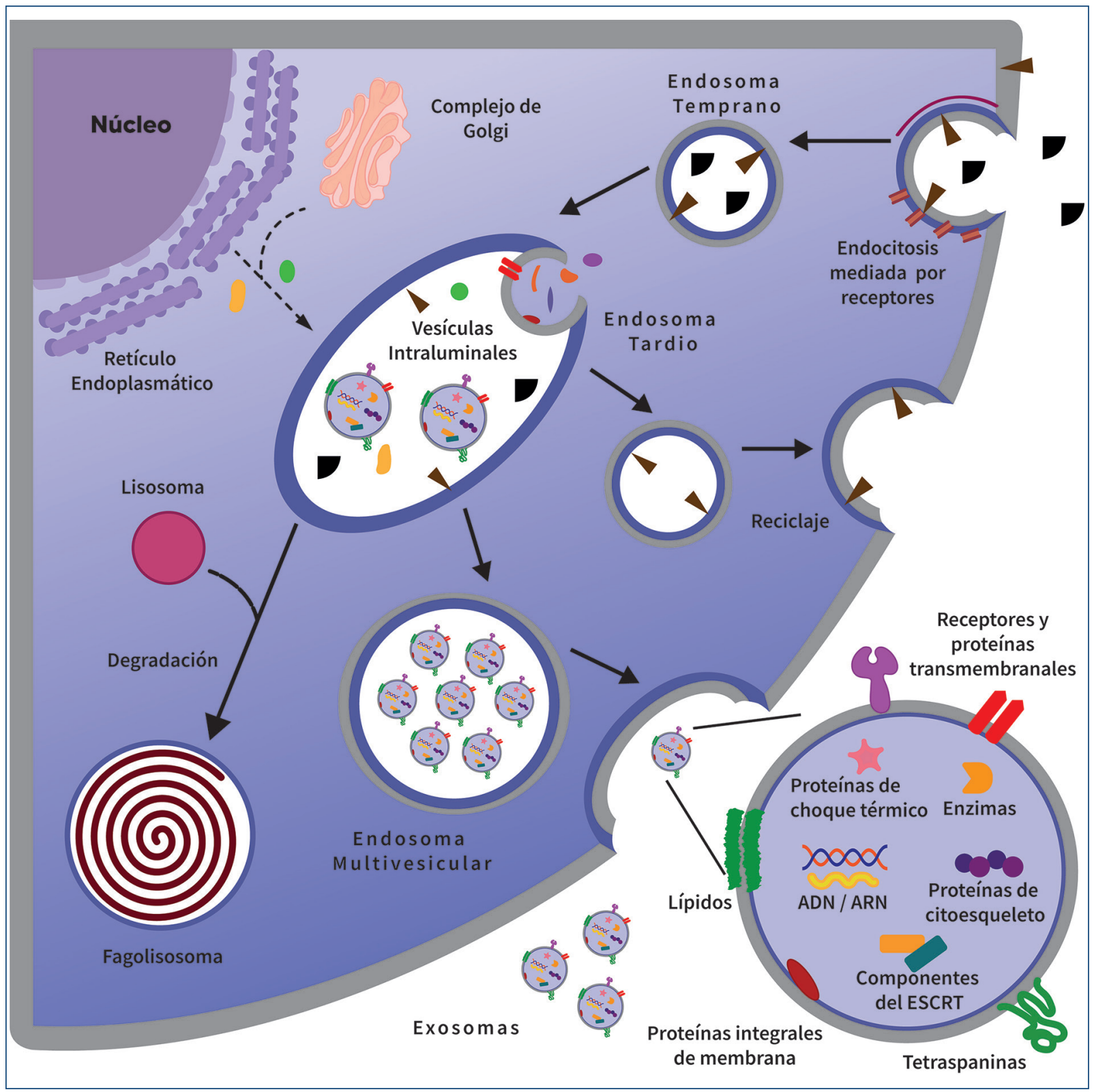

Figura 1. Sistema endomembranal implicado en la biogénesis exosomal. Los exosomas se generan a partir de la compartimentalización dinámica de la membrana plasmática (MP), que permite la internalización de macromoléculas, proteínas de superficie, ligandos y componentes celulares y que posteriormente son reciclados hacia la MP, degradados y/o excretados. La carga exosomal engloba diferentes clases de lípidos, material genético, enzimas, proteínas membranales y del citosol.

ESCRT: Endosomal sorting complex required for transport.

(tumor susceptibility 101) y la proteína ALIX, antígenos de superficie, proteínas inmunomoduladoras, proteasas y moléculas angiogénicas, entre otras ${ }^{28}$.

- Lípidos: independientemente de su origen celular, los EXO están enriquecidos en colesterol, esfingomielina y fosfolípidos, formando balsas lipídicas en su membrana ${ }^{29}$. Los lípidos son parte esencial de la estructura vesicular, por lo que se han detectado hasta 280 especies lipídicas exosomales ${ }^{30}$. Los EXO poseen mayor rigidez membranal comparado con la MP, ya que contienen más fosfolípidos desaturados y por una relación lípidos/proteínas elevada, por lo que no se degradan fácilmente y pueden circular de forma libre en los fluidos biológicos ${ }^{31}$. 
Tabla 1. Componentes exosomales comunes

\begin{tabular}{|c|c|c|c|c|}
\hline Tipo & Categoría & \multicolumn{2}{|c|}{ Ejemplos } & Ref. \\
\hline \multirow[t]{14}{*}{ Proteínas } & Tetraspaninas & \multicolumn{2}{|c|}{ CD9, CD37, CD53, CD55, CD63, CD81, CD82 } & 6,17 \\
\hline & $\begin{array}{l}\text { Proteínas de } \\
\text { citoesqueleto }\end{array}$ & \multicolumn{2}{|c|}{$\begin{array}{l}\text { Actina, tubulina, cofilina-1, moesina, miosina, vimentina, erzina, radixina, } \\
\text { perlecán, fibronectina, THBS1, IOGAP1, queratina }\end{array}$} & $6,17,18$ \\
\hline & De biogénesis & \multicolumn{2}{|c|}{ ESCRT-0, I, II, III, Her, Vps4, TSG101, alix, flotilina, clatrina } & 6 \\
\hline & De transporte y fusión & \multicolumn{2}{|c|}{$\begin{array}{l}\text { Proteína relacionada con RAS } 5 \text { y 7, anexinas I, II, IV, V, VI, dinamina, } \\
\text { sintaxina-3, RAB4, } 5,7 \text { y } 11 \text {, RAP1B, RABGDI, SLC3A2, CLIC1 }\end{array}$} & $6,17,18$ \\
\hline & $\begin{array}{l}\text { Proteínas de choque } \\
\text { térmico }\end{array}$ & \multicolumn{2}{|c|}{$\begin{array}{l}\text { aB-cristalina, HSP20, HSP22, HSP27, HSP40, HSP60, HSP70, HSP90, HSC70, } \\
\text { HSPA5, CCT2 }\end{array}$} & $6,18,19,20$ \\
\hline & De adhesión & \multicolumn{2}{|c|}{ ICAM-1, integrinas, lactaderina, MFGE8, P-selectina } & 6,17 \\
\hline & $\begin{array}{l}\text { Antígenos de } \\
\text { presentación }\end{array}$ & \multicolumn{2}{|c|}{ Antígeno leucocitario humano tipo I y II } & 6 \\
\hline & $\begin{array}{l}\text { Proteínas de } \\
\text { señalización }\end{array}$ & \multicolumn{2}{|c|}{$\begin{array}{l}\text { GTPasa, HRAS, sintenina-1, Gi2 } \alpha \text {, proteínas } 14-3-3 \text {, ARF1, CDC42, NRAS, } \\
\text { EHD1, EHD4, RAN, PEBP1, MIF, RRAS2, estomatina, PDCD6 }\end{array}$} & $6,17,18$ \\
\hline & De transporte & \multicolumn{2}{|c|}{ Receptor de transferrina } & 6 \\
\hline & Enzimas & \multicolumn{2}{|c|}{$\begin{array}{l}\text { Glucosa-6-fosfato isomerasa, ácido graso sintasa, gliceraldehído-3-fosfato } \\
\text { deshidrogenasa, PFKL, peroxiredoxina-1, hexocinasa, fosfoglicerato } \\
\text { cinasa-1, fosfoglicerato mutasa 1, piruvato cinasa, isoenzimas M1/M2, ATP } \\
\text { citrato liasa, ATPasa, glucosa-6-fosfato isomerasa, aspartato } \\
\text { aminotransferasa, aldehído reductasa, enolasa-1, lactato deshidrogenasa, } \\
\text { aldolasa-1, dipeptidil peptidasa-4 }\end{array}$} & $6,17,18,21$ \\
\hline & Citocinas & \multicolumn{2}{|c|}{ Factor de necrosis tumoral alfa } & 6 \\
\hline & Antiapoptosis & \multicolumn{2}{|c|}{ Alix, tiorredoxina peroxidasa } & 17 \\
\hline & $\begin{array}{l}\text { Reguladores de } \\
\text { transcripción }\end{array}$ & \multicolumn{2}{|c|}{ EEF1A1, EEF2, LGALS3, EEF1A2 } & 18 \\
\hline & Caveolares & \multicolumn{2}{|c|}{ Caveolina-1, caveolina-3 } & 22 \\
\hline \multirow[t]{6}{*}{ Lípidos } & Fosfolípidos & \multicolumn{2}{|c|}{$\begin{array}{l}\text { Fosfatidilcolina, fosfatidilserina, lisofosfatidilcolina, fosfatidiletanolamina, } \\
\text { fosfatidilinositol, ácido lisobifosfatídico }\end{array}$} & 23 \\
\hline & Colesterol & & & 23 \\
\hline & Ceramida & & & 14,23 \\
\hline & Esfingolípidos & \multicolumn{2}{|c|}{ Esfingomielina, hexosilceramida, lactosilceramida } & 17 \\
\hline & Gangliósidos & \multicolumn{2}{|c|}{ GM1, GM3 } & 24,25 \\
\hline & Otros & \multicolumn{2}{|c|}{$\begin{array}{l}\text { Acido araquidónico, prostaglandina E, 15-d prostaglandina J2, diacilglicerol, } \\
\text { triacilglicerol, hexadecilglicerol }\end{array}$} & 23,26 \\
\hline \multirow[t]{3}{*}{$\begin{array}{l}\text { Ácidos } \\
\text { nucleicos }\end{array}$} & ARN & miARN & $\begin{array}{l}\text { Let-7, miR-1, miR-15, miR--16, mi-17, mi-R-18, } \\
\text { miR-19-19b, miR-20, miR-21, miR-29a, miR-126, } \\
\text { miR-143, miR-145, miR-151, miR-155, miR-181, } \\
\text { miR-200, miR- 214, miR-320, miR- 375, miR-382 }\end{array}$ & 6,23 \\
\hline & & \multicolumn{2}{|c|}{ ARNm, ARN circ, ARNmit, ARNt, ARNsp, ARNp. } & 6,27 \\
\hline & ADN & \multicolumn{2}{|c|}{ ADNmt, ADNcs, ADNcd, ADN viral } & 27 \\
\hline
\end{tabular}

- Ácidos nucleicos: los EXO contienen ADN y ARN no codificantes; ADNcs, ADNcd, ARNm, ARNt, ARNcirc, ARNmt, ARNp; sin embargo, los miARN o ExomiRs ${ }^{32}$ son el tipo de ARN más abundante en EXO plasmáticos humanos ${ }^{6}$, incluso en proporciones superiores con respecto a su célula de origen, debido a mecanismos de selectividad que operan durante el empaquetamiento de la carga ${ }^{33}$. Actualmente sabemos que el transporte de miARN puede darse a través de lipoproteínas de alta densidad o por unión a 


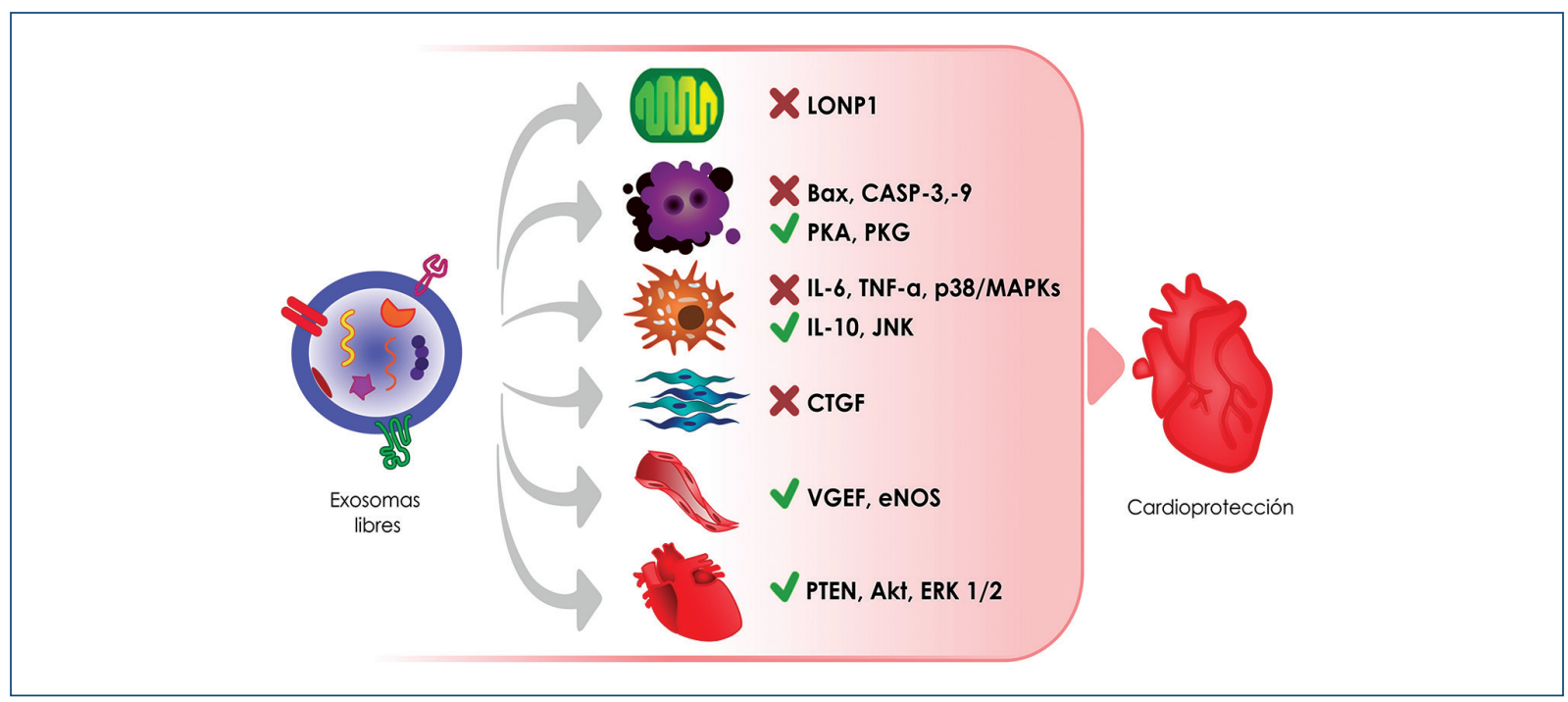

Figura 2. Efectos cardioprotectores regulados por exosomas. El contenido exosomal formado por múltiples biomoléculas desencadena un mensaje determinado en células y tejidos específicos, activando/inhibiendo vías de señalización que protegen la función cardiovascular. Entre estas vías destacan: la inhibición de LONP1, que mantiene la función mitocondrial; la inactivación de caspasas, vía Bax, que regulan procesos de apoptosis; el bloqueo de citocinas proinflamatorias (IL6, TNF- $\alpha$ ) y la vía p38/MAPK en células inmunitarias; la actividad antifibrótica por inhibición de CTGF; la angiogénesis mediada por VGEF y eNOS, y la activación de vías comunes de cardioprotección que incluyen a PTEN, Akt y ERK1/2. Akt: proteína cinasa B; Bax: proteína X asociada a Bcl-2; CASP 3 y 9: caspasas 3 y 9; CTGF: factor de crecimiento de tejido conectivo; eNOS: sintasa de óxido nítrico endotelial; ERK1/2: cinasa regulada por señal extracelular 1 y 2; IL 6 y 10: interleucinas 6 y 10; JNK: cinasas c-Jun N-terminal; LONP1: peptidasa Lon 1; MAPK: cinasa activada por mitógenos; PKA: proteína cinasa A; PKG: proteína cinasa G; PTEN: fosfatidilinositol- 3,4,5-trisfosfato 3-fosfatasa; TNF- $\alpha$ : factor de necrosis tumoral alfa; VGEF: factor de crecimiento endotelial vascular.

proteínas argonauta 2 y nucleofosmina $1^{34}$. Se ha reportado que los miARN exosomales están involucrados en procesos de angiogénesis, hematopoyesis y oncogénesis, y que algunos solo se expresan durante enfermedades cardiovasculares ${ }^{35}$. Las características lipídicas mencionadas anteriormente protegen al ADN y ARN de la degradación mediada por desoxirribonucleasas y ribonucleasas presentes en todos los fluidos corporales ${ }^{32}$.

\section{Relevancia del origen celular y del contenido de los exosomas en cardioprotección}

La composición de la carga exosomal se ha relacionado con la respuesta específica del tipo celular a diferentes estímulos ${ }^{36}$, al estado fisiológico o patológico de la célula donadora y a los mecanismos de biogénesis ${ }^{4}$. Por ejemplo, se ha observado que la administración de EXO séricos y plasmáticos no logra estimular la señalización protectora de ERK1/2 (extracelular signal-regulated kinase 1 y 2) sobre cardiomiocitos en condiciones de hiperglucemia, mientras que en normoglucemia se activa esta cinasa a través del receptor tipo toll-4 (TLR4) y HSP2 $2{ }^{37}$. Por otro lado, la aplicación de EXO purificados de plasma de ratas sometidas a acondicionamiento isquémico remoto disminuyen el tamaño del infarto en un modelo de daño por isquemia-reperfusión $(\mathrm{I} / \mathrm{R})^{38}$. De igual forma, las VE obtenidas del plasma de ratas sometidas a ejercicio mostraron efectos protectores contra la lesión por $\mathrm{l} / \mathrm{R}^{39}$. En cuanto al origen celular de estas estructuras, se ha reportado que los provenientes de líquido pericárdico mejoran la función vascular, estimulan la angiogénesis en modelos in vivo de isquemia, disminuyen la necrosis y aumentan la densidad vascular en la zona afectada ${ }^{10}$. Otros estudios han demostrado que los EXO de células mesenquimales troncales derivados de tejido adiposo disminuyen la necrosis y apoptosis en modelos in vivo e in vitro de $\mathrm{l} / \mathrm{R}$, tras la activación de la vía de señalización Wn$t / \beta$-catenina ${ }^{40}$ y que no solo los EXO derivados de tejido adiposo, sino también los producidos por médula ósea y cordón umbilical reducen la apoptosis y promueven la angiogénesis tras el infarto al miocardio ${ }^{41}$. En la figura 
2 se ilustra el efecto cardioprotector (directo o indirecto) de algunas estructuras y proteínas exosomales.

\section{Caveolas y caveolina}

La endocitosis mediada por caveolas es una ruta para la formación de EMV y finalmente de EXO. Las caveolas son invaginaciones de la MP consideradas como un subtipo de «balsas lipídicas", que resultan de las interacciones entre colesterol, esfingolípidos y esfingomielina ${ }^{42}$. Estos nanodominios de 50-100 nm se encuentran enriquecidos en diferentes tipos celulares, principalmente células endoteliales, fibroblastos, adipocitos y células musculares ${ }^{43}$. Las proteínas integrales de la caveola son las Cav: Cav- $1 \alpha$, Cav- $1 \beta$, Cav- $2 \alpha$, Cav-2 $\beta$ y Cav- $3^{44}$. Diversos estudios reportan que las caveolas, la Cav-1 y la Cav-3 son esenciales para la protección contra el daño por $\mathrm{I} / \mathrm{R}^{45}$. Se ha propuesto que las caveolas tienen un efecto mecanoprotector y que ante un aumento de las fuerzas hemodinámicas (gasto cardiaco), las caveolas presentes en endotelio pulmonar y músculo cardiaco sufren un aplanamiento, aumentando la superficie celular y liberando el exceso de tensión, protegiendo a las células de la ruptura celular $^{46}$. Por otro lado, si las caveolas se forman e internalizan tras el cierre membranal, pueden transportar diversas proteínas hacia blancos intracelulares, ya que poseen dominios de andamiaje para el anclaje y regulación de diversas moléculas regulatorias y de señalización ${ }^{47}$.

La Cav-1 es el principal componente estructural de la caveola se expresa predominantemente en células diferenciadas como células endoteliales, adipocitos, músculo liso y neumocitos tipo $1^{48}$. Se ha demostrado la importancia de la Cav-1 en cardioprotección, ya que atenúa la disfunción cardiaca inducida por neutrófilos ${ }^{49}$ y se sobreexpresa en terapias farmacológicas ${ }^{50}$. Por otro lado, Mierke, et al. sugieren una interacción coordinada de Cav-1 y la sintasa de óxido nítrico endotelial en la disminución de la capa íntima vascular, en la reestenosis y en la lesión del vaso en un modelo de trasplante vascular ${ }^{51}$. En modelos de isquemia cerebral y de extremidades, el efecto angiogénico de Cav-1 se ha relacionado con el factor de crecimiento endotelial vascular y su receptor ${ }^{52}$; con base en ello, sería interesante evaluar esta relación en la cardiopatía isquémica.

También se ha descrito la presencia de ARNm de Cav-1 en suero y plasma de pacientes con diferentes patologías y aunque la presencia de Cav-1 en el espacio extracelular ha sido poco estudiado, se relaciona con daño celular agudo inducido por hipoxia en algunos tejidos, particularmente en riñón ${ }^{53}$. Se sugiere que el desprendimiento de la Cav de la MP es resultado del daño hipóxico a la estructura caveolar. Por otro lado, la Cav-3 es una proteína presente únicamente en células musculares estriadas, donde regula la síntesis de óxido nítrico, la contracción del miocito y el metabolismo del colesterol $^{54}$. Feiner, et al. demostraron que los niveles de Cav-3 se reducen en un modelo murino de insuficiencia cardiaca izquierda y en insuficiencia cardiaca humana ${ }^{55}$. La Cav-3 es esencial para la protección cardiaca inducida por el receptor de opioides $\delta$ durante el preacondicionamiento ${ }^{56}$, mientras que la tolerancia a la I/R está asociada a la expresión de Cav- $3^{57}$. Durante el estrés cardiaco la autofagia regula la adaptación al daño celular, el cual parece depender de la Cav-3, logrando preservar la función mitocondrial y evitar la apoptosis ${ }^{58}$. Por otro lado, la Cav-3 interactúa con la adiponectina, formando un complejo AdipoR1/Cav-3, el cual confiere cardioprotección mediante la cinasa activada por monofosfato de adenina ${ }^{59}$. A la fecha no se ha reportado la presencia de Cav-3 en EXO de plasma humano.

\section{Proteínas de choque térmico}

Las HSP son moléculas ubicuas inducibles durante eventos de estrés celular, encargadas del plegamiento, ensamblaje, translocación y degradación de proteínas ${ }^{60}$. En EXO y VE se ha descrito que algunas isoformas de las HSP participan en la comunicación intercelular en condiciones de cardioprotección ${ }^{15}$. Por ejemplo, la HSP20 que se expresa en el músculo esquelético, cardiaco y liso, regula de forma transitoria la respuesta al estrés celular ${ }^{61}$. Se ha demostrado que al ser fosforilada por la proteína cinasa $A$ y por la proteína cinasa $G$, inhibe la actividad de caspasa-3 (CASP-3), disminuyendo la apoptosis $^{62}$. Los mismos autores sugieren que su efecto cardioprotector en un modelo de $\mathrm{I} / \mathrm{R}$ se relaciona con el bloqueo de la vía Bax (Bcl-2-associated X protein)/ CASP- $3^{63}$, por lo que su activación puede ser un punto clave de protección en la cardiopatía isquémica. También se la asocia con la reducción de la cardiotoxicidad en pacientes con cáncer tratados con doxorubicina ${ }^{64}$.

Las isoformas HSP60 y HSP70 se expresan en el tejido cardiaco; durante la hipoxia estas proteínas se desacoplan de Bax, permitiendo su translocación a las mitocondrias, donde al formar poros en la membrana favorecen la liberación de citocromo c, que en el citosol activará la vía de caspasas y la muerte por apoptosis. Existe también una forma de HSP60 mitocondrial, que evita la degradación de proteínas mitocondriales vía LONP1 (Lon peptidase 1). La disminución o deleción 
de HSP60 mitocondrial genera disfunción mitocondrial y una subsecuente dilatación e insuficiencia cardiaca ${ }^{65}$. El HSP60 de origen exosomal se une con el TLR4, promoviendo la liberación de factor de necrosis tumoral alfa (TNF- $\alpha$ ) e interleucina-6 (IL-6) ${ }^{65}$. También se ha reportado que la HSP70 extracelular puede ser útil para predecir la supervivencia de los pacientes con insuficiencia cardiaca, ya que el aumento de los niveles de HSP70 en pacientes con insuficiencia cardiaca se asocia con una mayor mortalidad ${ }^{66}$. Recientemente se ha documentado la sobreexpresión de HSP70 a nivel vascular en modelos de actividad física, en donde se previene la disfunción endotelial y se mantiene la musculatura vascular coronaria sana, capaz de proteger el miocardio ante insultos isquémicos ${ }^{67}$. Por otro lado, el consumo moderado de alcohol también se ha asociado con protección cardiaca postinfarto, y se sugiere que podría estar mediado por HSP70, hemooxigenasa 1 y superóxido dismutasa de manganeso, cuya sobreexpresión disminuye el tamaño del infarto y mejora la función ventricular ${ }^{68}$. Estos resultados correlacionan con los reportados por Guisasola, et al., quien demuestra que el consumo regular de alcohol aumenta los niveles de anticuerpos anti-HSP60, estimulando la producción de citocinas proinflamatorias y disminuyendo la cardioprotección ${ }^{69}$.

\section{Citocinas}

Las células inmunitarias participan en diversos eventos postisquémicos: regulan la eliminación de residuos celulares, favorecen la inflamación y estimulan la formación de tejido de cicatrización. Se ha reportado que los EXO de cardiomiocitos expuestos a isquemia estimulan la diferenciación de células dendríticas de médula ósea. Estas, a su vez, liberan EXO capaces de programar a las células $\mathrm{CD}^{+} \mathrm{T}$, que al secretar quimiocinas y mediadores inflamatorios contribuyen a mejorar la función cardiaca postinfarto ${ }^{70}$. También se sabe que miARN derivados de macrófagos promueven la inflamación cardiaca incrementando las IL-1 $\beta$ e IL-6, el TNF- $\alpha$ y el CCL2 (ligando de quimiocinas 2), y que específicamente los EXO derivados de macrófagos que contienen miR-155 disminuyen la proliferación de fibroblastos y aumentan la respuesta inflamatoria, lo que podría dañar los procesos de reparación tras el infarto al miocardio ${ }^{71}$. Estos datos enfatizan la dualidad de la respuesta inflamatoria y la necesidad de su oportuna regulación en el tiempo, ya que, en las fases tempranas del infarto, esta respuesta contribuye a mejorar la tasa de supervivencia promoviendo la reparación del tejido cardiaco; mientras que su descontrol lleva a la muerte celular y a la falla en el remodelaje cardiaco tras el aumento en la producción de especies reactivas de oxígeno, metaloproteinasas y citocinas proinflamatorias.

Es evidente el potencial terapéutico de estas y otras VE, pero también hay muchas preguntas que aún no se contestan en relación con la respuesta inflamatoria, por ejemplo: cuáles son los mecanismos que subyacen en el empacamiento y liberación de citocinas, si las formas asociadas a VE difieren de las formas libres en cuanto a función y si su aplicación alogénica o autóloga es segura en términos de inmunogenicidad en pacientes con enfermedades cardiovasculares.

\section{miARN exosomales}

Los miARN son una clase de ARN no codificante que controlan la expresión de algunos ARNm complementarios. Su presencia en suero y plasma sugiere que los miARN pueden cumplir funciones biológicas y genéticas en condiciones fisiopatológicas ${ }^{72}$. Particularmente, el estudio de miR-21 y su derivado miR-21-5p en EXO provenientes de células madre mesenquimales ha demostrado que tienen efectos paracrinos positivos en la supervivencia celular y en la angiogénesis, favoreciendo la recuperación del miocardio y la regeneración vascular posteriores al infarto del miocardio ${ }^{73}$. De forma importante, el miR-21 ejerce una regulación cardioprotectora a través de la vía de señalización PTEN/Akt (phosphatase and tensin homologue/protein kinase $B)^{73}$. En este tenor muchos más miARN se han asociado con la protección cardiaca ante eventos hipóxico/isquémicos, por lo que su estudio en EXO aportaría información útil sobre los mecanismos de cardioprotección.

\section{Conclusiones}

Cada vez existen más evidencias de que los EXO y otras VE, dependiendo de su origen celular y de los cambios en su contenido, tienen un efecto positivo o negativo en enfermedades cardiovasculares. El estudio de la biología exosomal y en particular de las moléculas que estas vesículas transportan, ayudará a definir nuevos candidatos a biomarcadores de daño cardiaco temprano o tardío, generando nuevas opciones diagnósticas, terapéuticas y pronósticas en el manejo de las patologías cardiovasculares. Sin embargo, ya que el entorno fisiopatológico en que se producen estas vesículas puede modificar su contenido, son indispensables más estudios preclínicos para conocer la 
composición específica de los EXO y los procesos que regulan su biogénesis, selección de carga, secreción, señalización, además de las vías la intercomunicación celular en enfermedades cardiovasculares.

Existen novedosas nanotecnologías con las que en un futuro próximo se podrían diseñar microvesículas en cantidades suficientes, que lleguen a blancos específicos y cuya carga tenga potencial terapéutico para realizar intervenciones de cardioprotección dirigidas e incluso personalizadas, así como para mejorar y/o producir un efecto sinérgico con las terapias cardioprotectoras existentes.

\section{Financiamiento}

La presente investigación no ha recibido ninguna beca específica de agencias de los sectores público, comercial, o sin ánimo de lucro.

\section{Conflicto de intereses}

Ninguno

\section{Responsabilidades éticas}

Protección de personas y animales. Los autores declaran que para esta investigación no se han realizado experimentos en seres humanos ni en animales.

Confidencialidad de los datos. Los autores declaran que en este artículo no aparecen datos de pacientes.

Derecho a la privacidad y consentimiento informado. Los autores declaran que en este artículo no aparecen datos de pacientes.

\section{Bibliografía}

1. Hutcheson JD, Aikawa E. Extracellular vesicles in cardiovascular homeostasis and disease. Curr Opin Cardiol. 2018;33(3):290-7.

2. Rezaie J, Rahbarghazi R, Pezeshki M, Mazhar M, Yekani F, Khaksar M, et al. Cardioprotective role of extracellular vesicles: A highlight on exosome beneficial effects in cardiovascular diseases. J Cell Physiol. 2019;234(12):21732-45

3. Davidson SM, Yellon DM. Exosomes and cardioprotection - A critical analysis. Mol Aspects Med. 2018r:60:104-14.

4. van Niel G, D'Angelo G, Raposo G. Shedding light on the cell biology of extracellular vesicles. Nat Rev Mol Cell Biol. 2018;19(4):213-28.

5. Théry $\mathrm{C}$, Amigorena $\mathrm{S}$, Raposo $\mathrm{G}$, Clayton A. Isolation and characterization of exosomes from cell culture supernatants and biological fluids. Curr Protoc Cell Biol. 2006; Chapter 3:Unit 3.22.

6. Zhang Y, Liu Y, Liu H, Tang WH. Exosomes: biogenesis, biologic function and clinical potential. Cell Biosci. 2019:9:19.

7. Wolfers J, Lozier A, Raposo G, Regnault A, Théry C, Masurier C, et al. Tumor-derived exosomes are a source of shared tumor rejection antigens for CTL cross-priming. Nat Med. 2001;7(3):297-303.

8. Lee Y, El Andaloussi S, Wood MJ. Exosomes and microvesicles: extracellular vesicles for genetic information transfer and gene therapy. Hum Mol Genet. 2012;21(R1):R125-34.
9. Raposo G, Stoorvogel W. Extracellular vesicles: exosomes, microvesicles, and friends. J Cell Biol. 2013;200(4):373-83.

10. Beltrami C, Besnier M, Shantikumar S, Shearn Al, Rajakaruna C, Laftah A, et al. Human pericardial fluid contains exosomes enriched with cardiovascular-expressed microRNAs and promotes therapeutic angiogenesis. Mol Ther. 2017;25(3):679-93.

11. Miaczynska M, Stenmark $H$. Mechanisms and functions of endocytosis. J Cell Biol. 2008;180(1):7-11.

12. Hessvik NP, Llorente A. Current knowledge on exosome biogenesis and release. Cell Mol Life Sci. 2018;75(2):193-208.

13. Elkin SR, Lakoduk AM, Schmid SL. Endocytic pathways and endosomal trafficking: a primer. Wien Med Wochenschr. 2016;166(7-8):196-204.

14. Elsherbini A, Bieberich E. Ceramide and exosomes: A novel target in cancer biology and therapy. Adv Cancer Res. 2018;140:121-54

15. Kowal J, Arras G, Colombo M, Jouve M, Morath JP, Primdal-Bengtson B, etal. Proteomic comparison defines novel markers to characterize heterogeneous populations of extracellular vesicle subtypes. Proc Natl Acad Sci U S A. 2016;113(8):E968-77.

16. Haraszti RA, Didiot MC, Sapp E, Leszyk J, Shaffer SA, Rockwell HE, et al. High-resolution proteomic and lipidomic analysis of exosomes and microvesicles from different cell sources. J Extracell Vesicles. 2016:5:32570.

17. Théry C, Zitvogel L, Amigorena S. Exosomes: composition, biogenesis and function. Nat Rev Immunol. 2002;2(8):569-79.

18. Mathivanan S, Ji H, Simpson RJ. Exosomes: extracellular organelles important in intercellular communication. J Proteomics. 2010;73(10):1907-20.

19. Reddy VS, Madala SK, Trinath J, Reddy GB. Extracellular small heat shock proteins: exosomal biogenesis and function. Cell Stress Chaperones. 2018;23(3):441-54

20. Wyciszkiewicz A, Kalinowska-Łyszczarz A, Nowakowski B, Kaźmierczak K, Osztynowicz K, Michalak S. Expression of small heat shock proteins in exosomes from patients with gynecologic cancers. Sci Rep. 2019;9(1):9817.

21. Göran Ronquist K. Extracellular vesicles and energy metabolism. Clin Chim Acta. 2019; 488:116-21.

22. Logozzi M, De Milito A, Lugini L, Borghi M, Calabrò L, Spada M, et al. High levels of exosomes expressing CD63 and caveolin-1 in plasma of melanoma patients. PLoS One. 2009;4(4):e5219.

23. Record M, Subra C, Silvente-Poirot S, Poirot M. Exosomes as intercellular signalosomes and pharmacological effectors. Biochem Pharmacol. 2011;81(10):1171-82.

24. Parolini I, Federici C, Raggi C, Lugini L, Palleschi S, De Milito A, et al. Microenvironmental $\mathrm{pH}$ is a key factor for exosome traffic in tumor cells. J Biol Chem. 2009;284(49):34211-22.

25. Yuyama K, Yamamoto N, Yanagisawa K. Accelerated release of exosome-associated GM1 ganglioside (GM1) by endocytic pathway abnormality: another putative pathway for GM1-induced amyloid fibril formation. J Neurochem. 2008;105(1):217-24.

26. Subra $C$, Grand $D$, Laulagnier $K$, Stella $A$, Lambeau $G$, Paillasse $M$, et al. Exosomes account for vesicle-mediated transcellular transport of activatable phospholipases and prostaglandins. J Lipid Res. 2010;51(8):2105-20.

27. Kalluri R, LeBleu VS. Discovery of double-stranded genomic DNA in circulating exosomes. Cold Spring Harb Symp Quant Biol. 2016;81:275-80.

28. Choi DS, Kim DK, Kim YK, Gho YS. Proteomics of extracellular vesicles: Exosomes and ectosomes. Mass Spectrom Rev. 2015;34(4):474-90.

29. Llorente $A$, Skotland $T$, Sylvänne $T$, Kauhanen D, Róg T, Orłowski A et al. Molecular lipidomics of exosomes released by PC-3 prostate cancer cells. Biochim Biophys Acta. 2013;1831(7):1302-9.

30. Skotland T, Sandvig K, Llorente A. Lipids in exosomes: Current knowledge and the way forward. Prog Lipid Res. 2017:66:30-41.

31. Record M, Silvente-Poirot S, Poirot M, Wakelam MJO. Extracellular vesicles: lipids as key components of their biogenesis and functions. J Lipid Res. 2018;59(8):1316-24.

32. Bhome R, Del Vecchio F, Lee GH, Bullock MD, Primrose JN, Sayan AE, et al. Exosomal microRNAs (exomiRs): Small molecules with a big role in cancer. Version 2. Cancer Lett. 2018;420:228-35.

33. Guduric-Fuchs J, O'Connor A, Camp B, O'Neill CL, Medina RJ, Simpson DA. Selective extracellular vesicle-mediated export of an overlapping set of microRNAs from multiple cell types. BMC Genomics. 2012;13:357.

34. Zhang J, Li S, Li L, Li M, Guo C, Yao J, et al. Exosome and exosomal microRNA: trafficking, sorting, and function. Genomics Proteomics Bioinformatics. 2015;13(1):17-24.

35. Khalyfa A, Gozal D. Exosomal miRNAs as potential biomarkers of cardiovascular risk in children. J Transl Med. 2014;12:162.

36. Villarroya-Beltri C, Baixauli F, Mittelbrunn M, Fernández-Delgado I, Torralba $\mathrm{D}$, Moreno-Gonzalo $\mathrm{O}$, et al. ISGylation controls exosome secretion by promoting lysosomal degradation of MVB proteins. Nat Commun. 2016;7:13588

37. Gartz M, Strande JL. Examining the paracrine effects of exosomes in cardiovascular disease and repair. J Am Heart Assoc. 2018;7(11):e007954.

38. Vicencio JM, Yellon DM, Sivaraman V, Das D, Boi-Doku C, et al. Plasma exosomes protect the myocardium from ischemia-reperfusion injury. $J$ Am Coll Cardiol. 2015;65(15):1525-36. 
39. Bei Y, Xu T, Lv D, Yu P, Xu J, Che L, et al. Exercise-induced circulating extracellular vesicles protect against cardiac ischemia-reperfusion injury. Basic Res Cardiol. 2017;112(4):38.

40. Cui X, He Z, Liang Z, Chen Z, Wang H, Zhang J. Exosomes from adipose-derived mesenchymal stem cells protect the myocardium against ischemia/reperfusion injury through Wnt/ $\beta$-catenin signaling pathway. Cardiovasc Pharmacol. 2017;70(4):225-31.

41. Xu H, Wang Z, Liu L, Zhang B, Li B. Exosomes derived from adipose tissue, bone marrow, and umbilical cord blood for cardioprotection after myocardial infarction. J Cell Biochem. 2020;121(3):2089-102.

42. Shaul PW, Anderson RG. Role of plasmalemmal caveolae in signal transduction. Am J Physiol. 1998;275(5):L843-51.

43. Kruglikov IL, Scherer PE. Caveolin-1 as a pathophysiological factor and target in psoriasis. NPJ Aging Mech Dis. 2019;5:4

44. Schwencke C, Braun-Dullaeus RC, Wunderlich C, Strasser RH. Caveolae and caveolin in transmembrane signaling: Implications for human disease. Cardiovasc Res. 2006;70(1):42-9.

45. Fridolfsson HN, Kawaraguchi $Y$, Ali SS, Panneerselvam M, Niesman IR Finley JC, et al. Mitochondria-localized caveolin in adaptation to cellular stress and injury. FASEB J. 2012;26(11):4637-49.

46. Cheng JP, Mendoza-Topaz C, Howard G, Chadwick J, Shvets E, Cowburn AS, et al. Caveolae protect endothelial cells from membrane rupture during increased cardiac output. J Cell Biol. 2015;211(1):53-61.

47. Patel HH, Head BP, Petersen HN, Niesman IR, Huang D, Gross GJ, et al. Protection of adult rat cardiac myocytes from ischemic cell death: role of caveolar microdomains and delta-opioid receptors. Am J Physiol Heart Circ Physiol. 2006;291(1):H344-50.

48. Sanon VP, Sawaki D, Mjaatvedt CH, Jourdan-Le Saux C. Myocardial tissue caveolae. Compr Physiol. 2015;5(2):871-86.

49. Young LH, Ikeda Y, Lefer AM. Caveolin-1 peptide exerts cardioprotective effects in myocardial ischemia-reperfusion via nitric oxide mechanism. Am J Physiol Heart Circ Physiol. 2001;280(6):H2489-95.

50. Patel HH, Tsutsumi YM, Head BP, Niesman IR, Jennings M, Horikawa Y, et al. Mechanisms of cardiac protection from ischemia/reperfusion injury: a role for caveolae and caveolin-1. FASEB J. 2007;21(7):1565-74.

51. Mierke J, Christoph M, Pfluecke C, Jellinghaus S, Wunderlich C Strasser $\mathrm{RH}$, et al. Atheroprotective role of caveolin-1 and eNOS in an innovative transplantation model is mainly mediated by local effects. Biochim Biophys Acta Mol Basis Dis. 2017;1863(2):529-36.

52. Gao Y, Zhao Y, Pan J, Yang L, Huang T, Feng X, et al. Treadmill exercise promotes angiogenesis in the ischemic penumbra of rat brains through caveolin-1/VEGF signaling pathways. Brain Res. 2014;1585:83-90.

53. Zager RA, Johnson A, Hanson S, de la Rosa V. Altered cholesterol localization and caveolin expression during the evolution of acute rena failure. Kidney Int. 2002;61(5):1674-83.

54. Sanon VP, Sawaki D, Mjaatvedt CH, Jourdan-Le Saux C. Myocardial tissue caveolae. Compr Physiol. 2015;5(2):871-86.

55. Feiner EC, Chung $P$, Jasmin JF, Zhang J, Whitaker-Menezes D, Myers $\mathrm{V}$, et al. Left ventricular dysfunction in murine models of hear failure and in failing human heart is associated with a selective decrease in the expression of caveolin-3. J Card Fail. 2011;17(3):253-63.

56. Tsutsumi YM, Kawaraguchi $Y$, Niesman IR, Patel HH, Roth DM. Opioid-induced preconditioning is dependent on caveolin-3 expression. Anesth Analg. 2010;111(5):1117-21.
57. See Hoe LE, Schilling JM, Tarbit E, Kiessling CJ, Busija AR, Niesman IR, et al. Sarcolemmal cholesterol and caveolin-3 dependence of cardiac function, ischemic tolerance, and opioidergic cardioprotection. Am J Physiol Heart Circ Physiol. 2014;307(6):H895-903.

58. Kassan A, Pham U, Nguyen Q, Reichelt ME, Cho E, Patel PM, et al. Caveolin-3 plays a critical role in autophagy after ischemia-reperfusion. Am J Physiol Cell Physiol. 2016;311(6):C854-65.

59. Wang $Y$, Wang X, Jasmin JF, Lau WB, Li R, Yuan Y, et al. Essential role of caveolin-3 in adiponectin signalsome formation and adiponectin cardioprotection. Arterioscler Thromb Vasc Biol. 2012;32(4):934-42.

60. Park CJ, Seo YS. Heat shock proteins: A review of the molecular chaperones for plant immunity. Plant Pathol J. 2015;31(4):323-33.

61. Martin TP, Currie S, Baillie GS. The cardioprotective role of small heatshock protein 20. Biochem Soc Trans. 2014:42(2):270-73.

62. Fan GC, Chu G, Mitton B, Song Q, Yuan Q, Kranias EG. Small heatshock protein Hsp20 phosphorylation inhibits beta-agonist-induced cardiac apoptosis. Circ Res. 2004;94(11):1474-82.

63. Fan GC, Ren X, Qian J, Yuan Q, Nicolaou P, Wang Y, et al. Nove cardioprotective role of a small heat-shock protein, Hsp20, against ischemia/reperfusion injury. Circulation. 2005;111(14):1792-9.

64. Fan GC, Zhou X, Wang X, Song G, Qian J, Nicolaou P, et al. Heat shock protein 20 interacting with phosphorylated Akt reduces doxorubicin-triggered oxidative stress and cardiotoxicity. Circ Res. 2008;103(11):1270-9.

65. Duan Y, Tang H, Mitchell-Silbaugh K, Fang X, Han Z, Ouyang K. Heat shock protein 60 in cardiovascular physiology and diseases. Front $\mathrm{Mol}$ Biosci. 2020;7:73.

66. Jenei ZM, Gombos T, Förhécz Z, Pozsonyi Z, Karádi I, Jánoskuti L, et al. Elevated extracellular HSP70 (HSPA1A) level as an independent prognostic marker of mortality in patients with heart failure. Cell Stress Chaperones. 2013;18(6):809-13.

67. Milne KJ, Wolff S, Noble EG. Myocardial accumulation and localization of the inducible $70-\mathrm{kDa}$ heat shock protein, Hsp70, following exercise. J Appl Physiol (1985). 2012;113(6):853-60.

68. Sato M, Fraga C, Das DK. Induction of the expression of cardioprotective proteins after mild-to-moderate consumption of alcohol. Pathophysiology. 2004;10(2):139-45.

69. Guisasola MC. Role of heat shock proteins in the cardioprotection of regular moderate alcohol consumption. Med Clin (Barc). 2016;146(7): 292-300.

70. Liu H, Gao W, Yuan J, Wu C, Yao K, Zhang L, at al. Exosomes derived from dendritic cells improve cardiac function via activation of CD4(+) T lymphocytes after myocardial infarction. J Mol Cell Cardiol. 2016;91: 123-33.

71. Wang C, Zhang C, Liu L, A X, Chen B, Li Y, et al. Macrophage-derived mir-155-containing exosomes suppress fibroblast proliferation and promote fibroblast inflammation during cardiac injury. Mol Ther. 2017;25(1):192-204

72. Vaka VR, McMaster KM, Cornelius DC, Ibrahim T, Jayaram A, Usry N, et al. Natural killer cells contribute to mitochondrial dysfunction in response to placental ischemia in reduced uterine perfusion pressure rats. Am J Physiol Regul Integr Comp Physiol. 2019;316(5):R441-7.

73. Wang K, Jiang Z, Webster KA, Chen J, Hu H, Zhou Y, et al. Enhanced cardioprotection by human endometrium mesenchymal stem cells driven by exosomal microRNA-21. Stem Cells Transl Med. 2017;6(1):209-22. 